\title{
Power quality analysis in a distribution network of a quarry processing plant
}

\author{
Joseph Cudjoe Attachie*, Christian Kwaku Amuzuvi \\ Department of Electrical and Electronic Engineering, University of Mines and Technology, Tarkwa, Ghana
}

Email address:

jattachie@yahoo.com(J. C. Attachie),jcattachie@umat.edu.gh(J. C. Attachie)

\section{To cite this article:}

Joseph Cudjoe Attachie, Christian Kwaku Amuzuvi. Power Quality Analysis in a Distribution Network of a Quarry Processing Plant. Journal of Electrical and Electronic Engineering. Vol. 1, No. 4, 2013, pp. 90-94. doi: 10.11648/j.jeee.20130104.15

\begin{abstract}
Power quality problems encompass a wide range of different phenomena. Each of these phenomena may have a variety of different causes and different solutions that can be used to improve upon the power quality and equipment performance. This paper analyses the malfunctioning of a capacitor bank at a quarry processing company (QPC) in the Western Region of Ghana. The QPC had held the Electricity Company of Ghana (ECG) responsible for the damage. According to QPC, the damage was due to frequent power fluctuations and outages experienced from the ECG distribution network. The paper presents and discusses techniques used to investigate the cause of the problem. The result of the analysis of the damage was conducted using the electromagnetic transient programme (EMTP) and relates the damage to harmonic resonance produced from the QPC power distribution network. It is recommended that, for reliable, safe and economic operation of capacitor banks, the harmonic content of an electrical installation is measured and analyzed before installation.
\end{abstract}

Keywords: Power Quality, Capacitor Bank, Harmonic Resonance, Resonant Frequency, Distribution Network

\section{Introduction}

Power quality encompasses voltage, frequency and waveform distortions that result in failure of customer equipment. From a theoretical point of view, good power quality can be taken to mean that: the voltage supplied by the utility at the customer's service entrance is steady and within the prescribed range; that the ac frequency is steady and very close to its nominal value (within a fraction of a percent); and that the waveform or shape of the voltage curve versus time very much resembles the smooth sine wave (a condition also described as the absence of harmonic distortion). In practice, however, it makes more sense to consider power quality as the compatibility between what comes out of an electric outlet and the load that is plugged into it.

Over recent years, there has been a significant increase in the installation and use of power electronic equipment in electrical power distribution systems. The operation of this equipment has in many cases significantly affected the electrical power quality to such an extent that measures have to be implemented in order to minimise the resultant adverse effects on the electrical plant and equipment [1].

Distortion of sinusoidal voltage and current waveforms caused by harmonics is one of the major power quality concerns in the electric power industry. Considerable efforts have been made in recent years to improve the management of harmonic distortions in power systems. Standards for harmonic control have been established. Instruments for harmonic measurements are widely available. The area of power system harmonic analysis has also experienced significant advancement [2-3].

During the process of power quality measurement, the electric utility providers use class A (Medium Voltage (MV) bus) and class B (Low Voltage (LV) bus) equipment. Power quality parameters include: voltage frequency, RMS voltage values, voltage flicker, imbalance in the voltage three phase system and voltage harmonic distortion.

Furthermore, the electric utility suppliers measure the magnitude of all voltage dips or sags that occur in the networks, as well as swells and interruptions both in number and duration. Power quality is ultimately a consumer-driven issue, and the end user's point of reference takes precedence.

The objective of this paper is to investigate the frequent failure of a capacitor bank at a QPC in the Western Region of Ghana. The company blamed the ECG for damaging its power factor correction capacitor bank. According to the company, the cause of the damage was due to frequent power fluctuations and outages experienced from the ECG 
distribution network. The QPC are therefore asking the ECG to pay for the replacement of the capacitor bank and also restrain ECG from surcharging them for operating at a low power factor.

\section{Materials and Methods}

\subsection{Resonant Harmonic}

The resonant frequency for a particular inductance/capacitance combination can be computed from a variety of different formulae depending on what data are available. The basic resonant frequency equation is:

$$
\mathrm{f}_{\mathrm{r}}=\frac{1}{2 \pi \sqrt{\mathrm{LC}}}
$$

Power systems analysts typically do not have L and C readily available, so it is commonly computed through the resonant harmonic, $h_{r}$, based on fundamental frequency impedances and ratings [4]:

$$
\mathrm{h}_{\mathrm{r}}=\sqrt{\frac{\mathrm{MVA}_{\mathrm{SC}}}{\mathrm{Mvar}_{\text {cap }}}}
$$

where, $\mathrm{h}_{\mathrm{r}}=$ resonance harmonic, $\mathrm{MVA}_{\mathrm{SC}}=$ system short-circuit in MVA and Mvar $_{\text {cap }}=$ Mvar rating of capacitor bank.

However, at a secondary side of a transformer, resonant frequency of a network can be determined using the relation [5]:

$$
\mathrm{h}=\sqrt{\frac{\mathrm{kVA}_{\text {transformer }}}{\mathrm{Z}_{\text {transformer }} \times \mathrm{kvar}}}
$$

where, $h$ is harmonic frequency in per unit, $Z$ is the impedance of the transformer in percent, $k V A$ is the power rating and kvar is the reactive power rating of the capacitor bank.

\subsection{Network Configuration}

The equivalent representation of the power distribution network of the company is depicted in Fig. 1. The source is represented as a Thevenin equivalent with an $\mathrm{X} / \mathrm{R}$ value of 5.3. The short circuit power is $1800 \mathrm{MVA}$ at a voltage of 33 $\mathrm{kV}$ as base. The $2 \mathrm{MVA}, 33 / 0.433 \mathrm{kV}$ transformer at the station is represented by a $6 \%$ impedance. The active power (P) and reactive power (Q) load of the station are $390 \mathrm{~kW}$ and 547 kvar respectively.

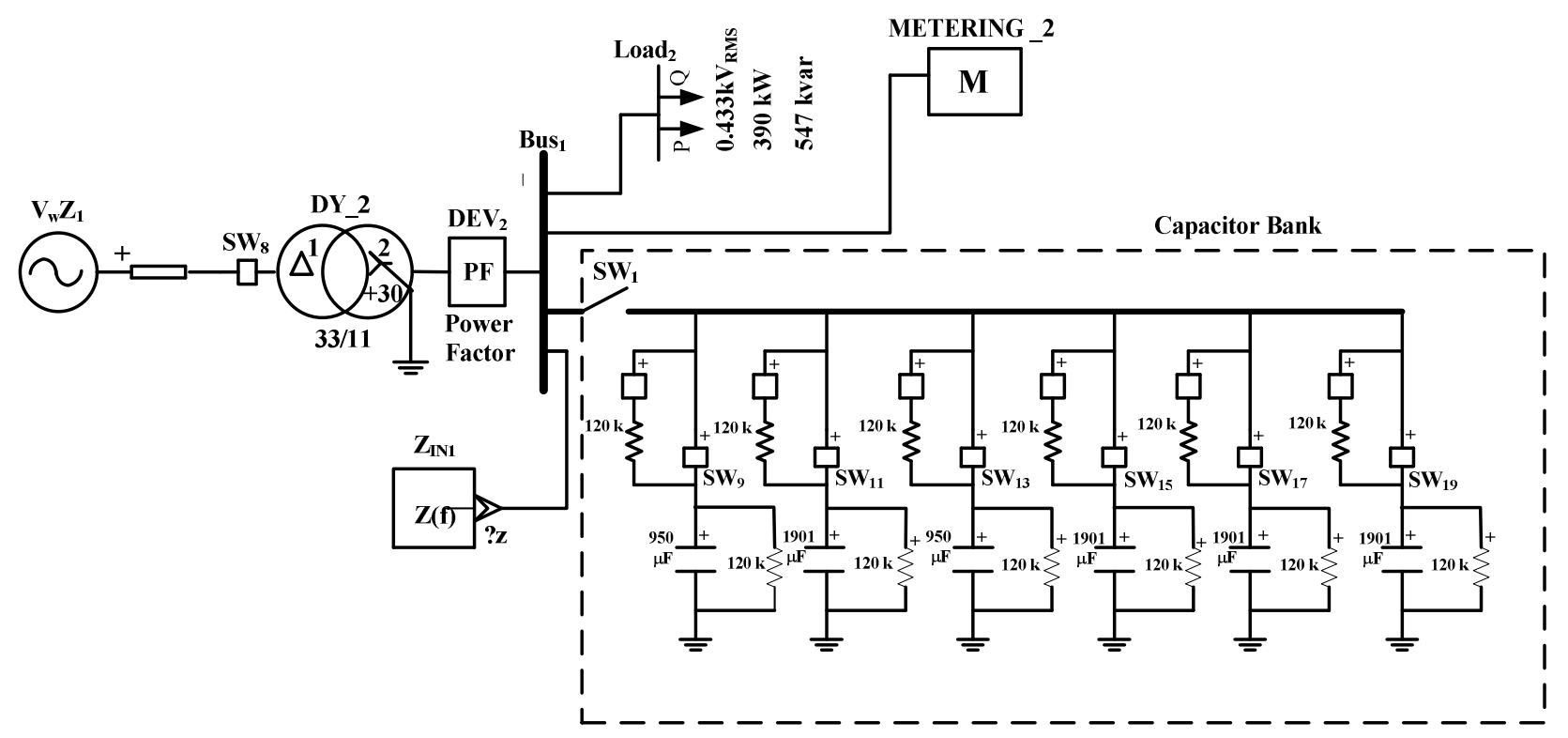

Figure 1. A single line diagram of the QPC distribution network.

The PQ loads are primarily ac motors with variable speed drives. These drives are occasionally brought into operation as and when the system demands it. On the average, the 2 MVA transformer is about 30\% load with a maximum load of $630 \mathrm{kVA}$. The only significant harmonic sources on the bus are the drives.

The capacitor bank contains six steps of varying kvars. Step 1 and 3 are each 56 kvar. The rest of the steps are each 112 kvar. The combined kvars of the six steps is 560 kvar. All of the kvar ratings are at a maximum voltage of $750 \mathrm{~V}$.
None of the steps are configured as harmonic filters. Each of the steps is protected by a 160 A current limiting fuse. The steps switch in and out of service automatically based on the power factor correction control algorithm in the bank.

\subsection{Power Quality Measurements}

In order to measure the power quality at the company, the Megger power analyzer, model PA-9 Plus, was used. The power quality at the premises was monitored for a pe- 
riod of five days. The main electrical parameters tracked included power frequency, harmonics, transients and voltage fluctuations. From the power quality measurements, transient overvoltages were not found. Accordingly, our discussions on the electrical parameters were limited to power frequency, harmonics and voltage fluctuations.

\subsection{Effect of Power Interruptions}

Frequent power interruption was one of the factors the company cited as being responsible for the capacitor failure. As a result, effect of power interruption on the capacitor bank was examined. This was done by simulation using EMTP engineering software.

\subsection{Outage Statistics}

On the day the capacitor bank failed, six successive outages were recorded within a minute, followed by a loud explosion at their substation. To investigate this claim, statistical record of outages on the feeder serving the company was obtained and studied.

\section{Results and Discussion}

\subsection{Voltage Fluctuation}

Voltage limits are based on overall permissible voltage range allowable under standards. ECG operating voltage (in low voltage network) ranges from a maximum of $253 \mathrm{~V}$ to a minimum of $207 \mathrm{~V}$. This is based on a nominal voltage of $230 \mathrm{~V}$ representing $\pm 10 \%$. Fig. 2 shows voltage envelope as measured by the power analyzer. Operating voltage range of 235 to $242 \mathrm{~V}$ was recorded. This represents a voltage variation of $2-5 \%$ in comparison to ECG nominal voltage. The variation was therefore within the acceptable operating voltage limits.

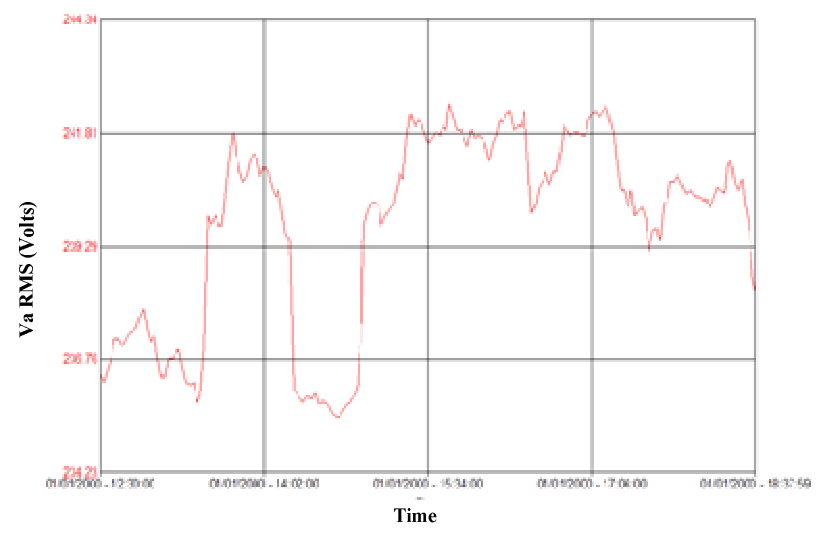

Figure 2. Voltage envelope as measured by the power analyzer.

\subsection{Frequency}

The term power frequency disturbance describes events that are slower and long lasting compared to electrical tran- sients. Power frequency disturbance can be harmful to electrical equipment [6]. The extent of damage varies from one piece of equipment to another and depends on the extent of the disturbance. One of the most common power frequency disturbances is voltage sag. From Fig. 3, frequency variation of about $0.5 \%$ was noted. Effect of this variation reflected as $2 \%$ voltage sag in Fig. 2 . The $2 \%$ voltage variation was within the tolerable and acceptable ECG operating voltage limit.

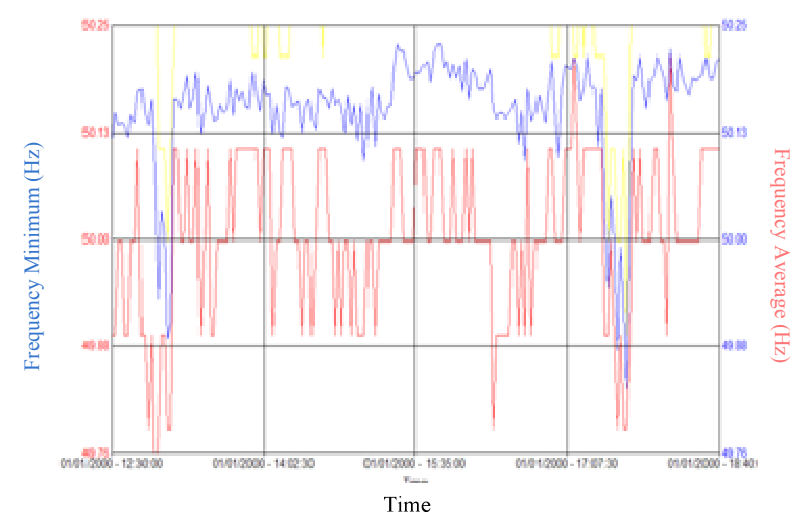

Figure 3. Frequency graph as measured.

\subsection{Harmonic Measurement}

Harmonics is one of the possible causes of capacitor bank failures and fuse operations [7]. Measurements were performed to quantify the harmonic voltages and currents in the capacitors in order to study whether harmonics were the cause of the failures. Result of the harmonic measurement at full load of the QPC is shown in Fig. 4

The natural (resonant) frequencies of the QPC network computed at the respective steps of the capacitor bank are shown in Table 1. It is obvious that, the natural frequency of the network changes with the steps of the capacitor bank.

Table 1. Natural frequencies of the QPC network at respective steps of the capacitor bank

\begin{tabular}{lllllll}
\hline & Step1 & Step2 & Step3 & Step4 & Step5 & Step6 \\
\hline kvar & 56 & 168 & 224 & 336 & 448 & 560 \\
$\begin{array}{l}\text { Resonant } \\
\text { Frequency(Hz) }\end{array}$ & 26 & 15 & 13 & 10 & 9 & 8 \\
\hline \multicolumn{5}{c}{ Measured harmonic current (A), refer to Fig. 3 } \\
\hline 0 & 11.4 & 4.2 & 0 & 22.7 & 0 \\
\hline
\end{tabular}

Table 1 shows that the network is switched into resonance with step 2, 3 and (or) 5 engaged. This is because the natural frequency of the network at these steps (i.e. resonant steps) coincides with the 15th, 13th and 9th harmonic content of the network. The effect of the resonant steps on the capacitor bank was examined by simulation using the EMTP software. 


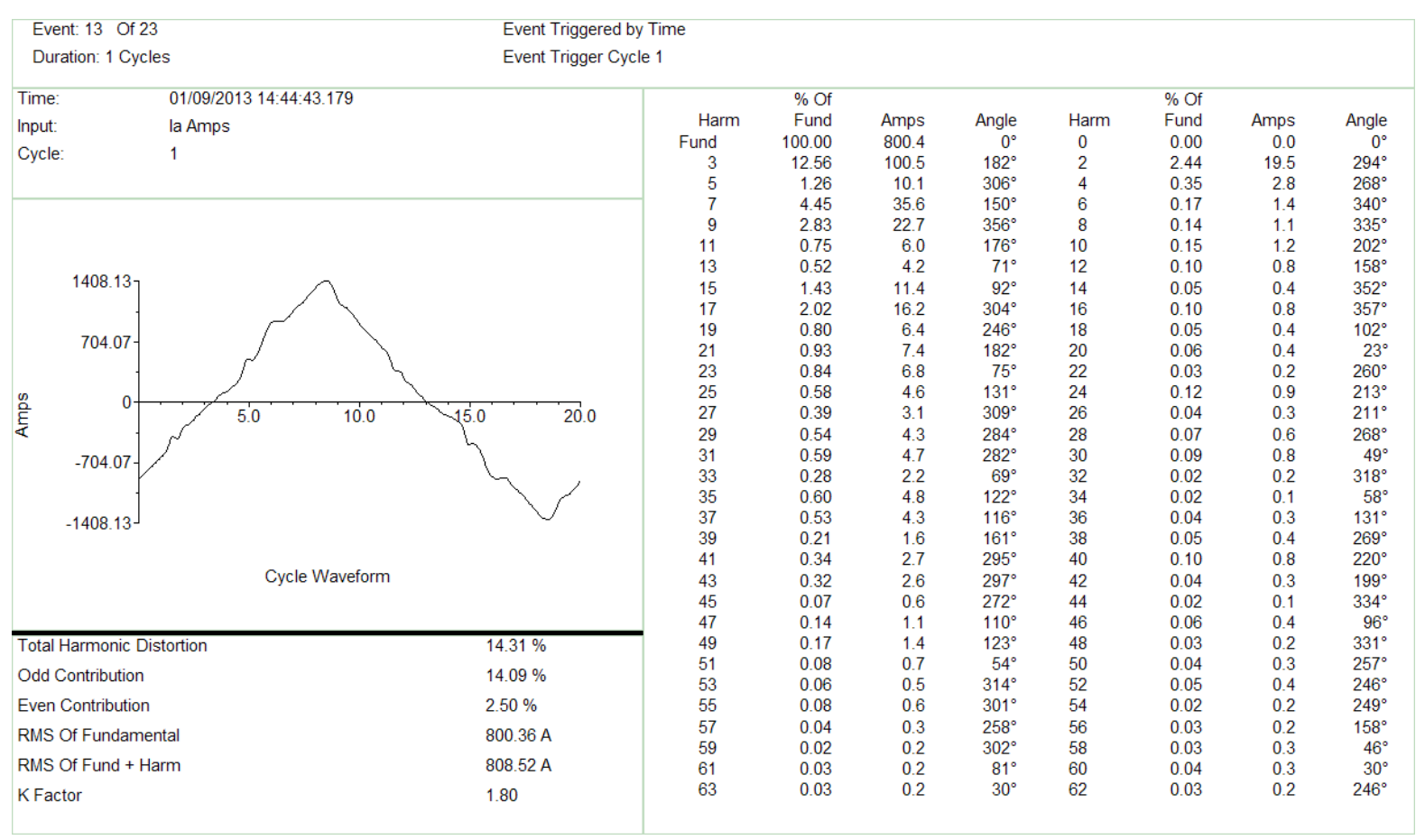

Figure 4. Harmonic measurement.

Harmonic currents of 11.4, 4.2 and 22.7 A associated with the 15, 13 and 9th harmonic orders were in turn injected into the network. Voltage at the LV bus and current drawn by the respective steps were observed. The worst resonance occurred at engagement of step-5. However, the effect of the resonance was more pronounced in step-3 capacitor bank. In Fig. 5, current amplification of about 170\% of the normal current was observed in step-3 capacitor bank. This was about 160 A drawn by step 3 capacitor bank: just equal to the size of the associated protecting fuse. The IEEE standard 18-2002 allows $135 \%$ of capacitor nominal load but this is intended for contingencies and not intended for normal continuous load. This means that, the magnitude of the current amplification is destructive.
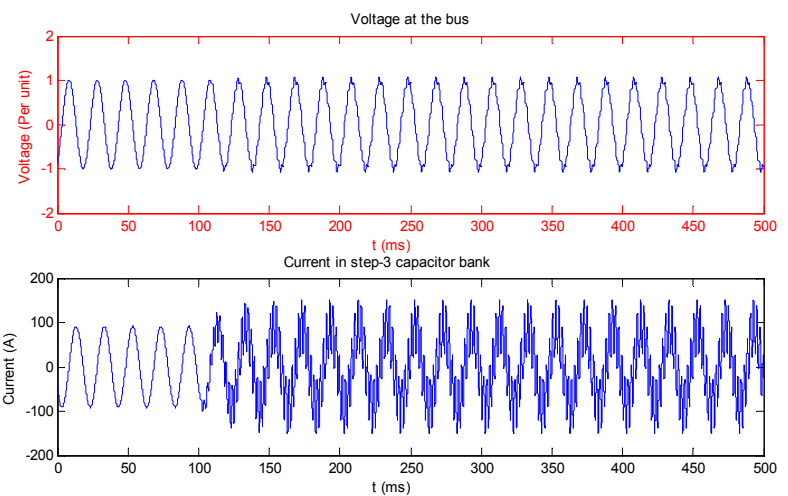

Figure 5. Voltage at bus-1 and current in step-3 capacitor bank.

Generally, a fuse will start fusing from 125 to $165 \%$ of its rated current [8]. Therefore, it was expected that, the 160 A current limiting fuse of the capacitor bank should operate from 200 to 264 A. This means that, the 160 A fuse would not blow under the resonance condition. The capacitor bank will therefore be expected to get damaged before the fuse blows.

Furthermore, capacitor fuses are deliberately oversized to take care of capacitor switching transient and prevent excessive nuisance blow-outs [5]. Although the fuse may blow at certain resonance condition, capacitor fuses are not primarily sized to take care of resonance conditions. It is therefore important to measure and analyse harmonic contents of an electrical installation and take preventive decision before installing a capacitor bank.

\subsection{Power Interruptions}

The effect of power interruptions on the capacitor bank was examined. Overvoltage and inrush currents in the capacitor bank during restoration of power were studied. Fig. 6 depicts that, overvoltage induced from the power restoration was 1.5 per unit and this lasted for $1.8 \mathrm{~ms}$. The IEEE standard 18-1992 and IEEE standard 1036-1992 allow overvoltage of 1.7 per unit for 1 second. It is obvious that the overvoltage induced by the power restoration was well within the standard safety limits and therefore, should have no effect on the capacitor bank.

Fig. 7 shows inrush current drawn by step 2 and step 3 capacitor banks. Step 2 and step 3 give a representative result because as noted earlier, they are made up of 112 and 56 kvar respectively. The inrush currents drawn by step 2 and step 3 capacitor banks were 1342 A and 623 A respectively. The IEC 60871-1 standard establishes that the peak value of the overcurrent due to switching operations should be limited to a maximum of $100 \times \mathrm{IN}$ [9], where $\mathrm{IN}$ is the rms value of the nominal current. By this standard, the maximum inrush current limit for the 112 kvar and 56 kvar 
are 19600 A and 9100 A respectively. Consequently, it can be seen that the current drawn by the capacitors during the power restoration were far less compared with their maximum inrush current limits.

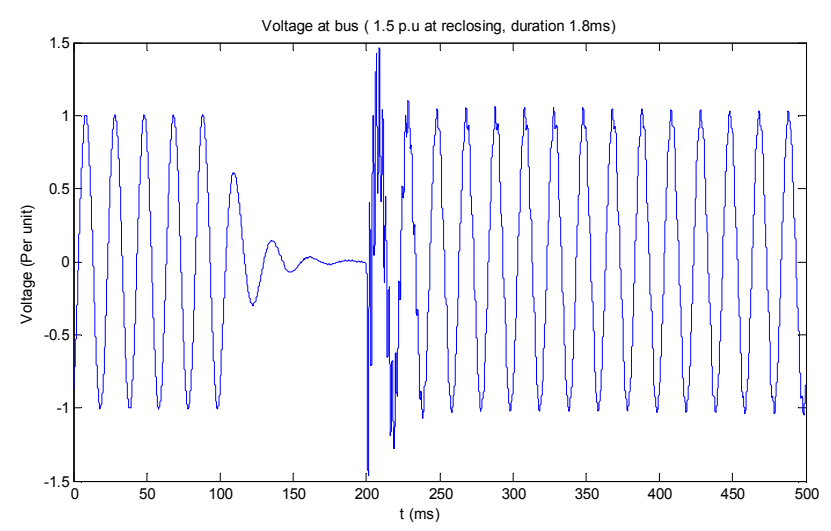

Figure 6. Overvoltage resulting from power restoration.
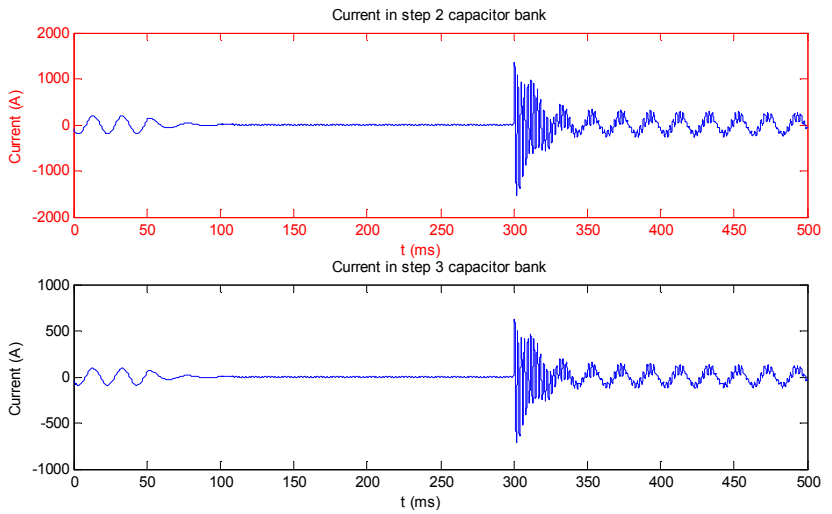

Figure 7. Inrush currents in the capacitor bank as a result of power restoration

\section{Conclusion}

The voltage operating envelope measured at the QPC substation ranged between 2 to $5 \%$ of the nominal voltage. After the power had been restored, transient overvoltage of 1.5 per unit occurred and lasted for $1.8 \mathrm{~ms}$. This overvoltage was within the standard safety regulation. The capacitor bank was not affected by power fluctuation. It was noted that, harmonic study was not conducted before the capacitor bank installation. The total harmonic distortion being produced by the company is $15 \%$ which exceeds ECG standard limit of 5\% required from customers. The analysis revealed that, the problem relating the capacitor damage was actually caused by high harmonic current produced by a 9th harmonic order which emanated from the capacitor bank on the LV busbar.

\section{Acknowledgements}

The authors wish to express their profound gratitude to George Eduful and Kivlyn N. Asante both of the Electricity Company of Ghana for their immense contribution to this research.

\section{References}

[1] ABS (2006), Guidance Notes on Control of Harmonics in Electrical Power Systems Houston, USA, 2006.

[2] Task Force on Harmonics Modeling and Simulation, "The Modeling and Simulation of the Propagation of Harmonics in Electric Power Networks Part I: Concepts, Models and Simulation Techniques", IEEE Transactions on Power Delivery, Vol.11, No.1, January 1996, pp. 452-465.

[3] Task Force on Harmonics Modeling and Simulation, "The Modeling and Simulation of the Propagation of Harmonics in Electric Power Networks Part II: Sample Systems and Examples", IEEE Transactions on Power Delivery, Vol.11, No.1, January 1996, pp. 466-474.

[4] R. Dugan, Electric Power Systems Quality, Mc Graw-Will, EUA, 2003, pp. 167-224.

[5] T. Blooming, "Capacitor Application Issues", IEEE Transaction on Industry Applications, vol. 44, no. 4, 2008, pp. $1013-1026$.

[6] C. Sankaran, Power Frequency Disturbance, Online source. Available at www.crenetbase.com/doi/pdf/10.1201/9781420041026.ch2: CRC Press LLC, 2002.

[7] M. B. P. Thomas, "Capacitor Failure Analysis: A Troubleshooting Case Study", Online source, date accessed 5th January, 2013. Available at www.asocem.org.pe/bivi/sa/dit/icem/05_04-2005.pdf, 2005.

[8] S. A. Ali, "Capacitor Banks Switching Transients in Power Systems", Energy Science and Technology, Vol. 2 , No.2, 2011, pp. 62-73.

[9] E. Issouribehere, "Measurements and Studies of Harmonics and Switching Transient in Large HV Shunt Capacitor Bank", IEEE Power Engineering Society General Meeting, 2007. 